\title{
The Pizzagame: A virtual public goods game to assess cooperative behavior in children and adolescents
}

\author{
Jan Keil $^{1}$ - Andrea Michel ${ }^{1,2}$ - Fabio Sticca ${ }^{3}$ - Kristina Leipold ${ }^{4}$ - Annette M. Klein ${ }^{1}$. \\ Susan Sierau $^{1} \cdot$ Kai von Klitzing $^{1} \cdot$ Lars O. White $^{1}$
}

Published online: 7 September 2016

(C) Psychonomic Society, Inc. 2016

\begin{abstract}
Social dilemmas are characterized by conflicts between immediate self-interest and long-term collective goals. Although such conflicts lie at the heart of various challenging social interactions, we know little about how cooperation in these situations develops. To extend work on social dilemmas to child and adolescent samples, we developed an ageappropriate computer task (the Pizzagame) with the structural features of a public goods game (PGG). We administered the Pizzagame to a sample of 191 children 9 to 16 years of age. Subjects were led to believe they were playing the game over the Internet with three sets of two same-aged, same-sex coplayers. In fact, the co-players were computer-generated and programmed to expose children to three consecutive conditions: (1) a cooperative strategy, (2) a selfish strategy, and (3) divergent cooperative-selfish strategies. Supporting the validity of the Pizzagame, our results revealed that children and adolescents displayed conditional cooperation, such that their contributions rose with the increasing cooperativeness of their co-players. Age and gender did not influence children and adolescents' cooperative behavior within each condition.
\end{abstract}

Jan Keil

jan.keil@medizin.uni-leipzig.de

1 Department of Child and Adolescent Psychiatry, Psychotherapy and Psychosomatics, University of Leipzig, Liebigstraße 20a, 04103 Leipzig, Germany

2 Department of Developmental Psychology, Friedrich-Schiller-University of Jena, Jena, Germany

3 Department of Empirical Educational Research, University of Konstanz/Thurgau University of Teacher Education, Konstanz, Germany

4 Faculty of Business and Economics, Dresden University of Technology, Dresden, Germany
However, older children adapted their behavior more flexibly between conditions to parallel the strategies of their coplayers. These results support the utility of the Pizzagame as a feasible, reliable, and valid instrument for assessing and quantifying child and adolescent cooperative behavior. Moreover, these findings extend previous work showing that age influences cooperative behavior in the PGG.

Keywords Public goods game $\cdot$ Cooperation $\cdot$ Prosocial behavior $\cdot$ Computerized task $\cdot$ Child development

We can choose to pay our taxes, organize a reunion with our classmates, prepare a meal with the family, or clean up with friends after a party, but we can also rely on others to do the work and reap the benefits from their efforts without contributing. Such choices make up the fabric of our daily interpersonal interactions, many of which might be construed as social dilemmas. In these situations, immediate self-interest runs contrary to potential long-term collective gains (Van Lange, Joireman, Parks, \& van Dijk, 2013). Interestingly, humans quite successfully manage to cooperate in such situations, which has enabled many of the accomplishments of modern societies, including government, health care, or accessible education (De Dreu, 2013).

Numerous theories state reasons why and when cooperation emerges in social dilemmas (for an overview, see Parks, Joireman, \& Van Lange, 2013). However, despite substantial work on adults, we know relatively little about how the propensity to cooperate in these situations develops. Additionally, the few existing studies with children have produced inconsistent empirical results and contain several methodological drawbacks. Therefore, with the help of a novel, computerized, and developmentally appropriate 
instrument, we aimed to overcome some of these drawbacks and promote our understanding of how cooperation develops in children and adolescents.

\section{The public goods game}

A classic example of a social dilemma is the public goods game (PGG; Hardin, 1968). Public goods refer to resources available to, and consumable by all group members, irrespective of how much an individual contributes to their provision (Olson, 1965), such as a clean environment or public services (Gummerum, Hanoch, \& Keller, 2008). Groups achieve their social optimum if everyone chooses to contribute to the public good and thus "pulls his/her weight," but the individual profits most by choosing a selfish strategy (i.e., freeriding) and exploiting others (Dawes, 1980). Due to this conflict, inherent in ample social interactions, these social dilemmas are especially appealing for studying cooperation in groups (Parks et al., 2013).

In the corresponding laboratory situation of a PGG, players receive an initial endowment of a resource and must then decide how much they want to keep for themselves or pool toward a public good. The latter is subsequently multiplied by a set factor and equally redistributed among all group members. Importantly, paralleling other social dilemmas, the outcome for an individual in this situation does not only depend on what he/she does but also on what everyone else does (strategic interdependency; Gross \& Heinrichs, 2010).

Although standard rational choice assumptions suggest that individuals would opt for the selfish strategy, various experiments have demonstrated that individuals behave much more cooperatively (Colman, 2003). Thus, they contribute a considerable amount in the first round (40\%-60\% of their resources), but contributions steadily dwindle thereafter. Freeriding paired with the tendency for conditional cooperation (i.e., cooperate if others also cooperate) is thought to partly account for this pattern (Fischbacher, Gächter, \& Fehr, 2001).

\section{Age and gender differences}

Despite extensive experimental research with adults in PGGs (for reviews, see Chaudhuri, 2011; Ledyard, 1995; Zelmer, 2003), few studies to date have examined child or adolescent cooperative behavior in these situations. Previous work has shown that 5-year-olds playing a simplified PGG begin to adopt conditionally cooperative strategies (Vogelsang, Jensen, Kirschner, Tennie, \& Tomasello, 2014). Moreover, an experimental study on 5- to 12-year-olds suggests that they initially contribute about the same share as adults, but then increase contributions, which subsequently plateau and finally decline (Harbaugh \& Krause, 2000). This curvilinear trajectory diverges from the continuous decrease in adults. However, inconsistent results and methodological drawbacks limit the conclusions that we can draw from previous developmental work, in the following ways.

First, studies analyzing the influence of age on cooperative behavior have yielded inconsistent results. Although two studies reported that older children showed more cooperative behavior by contributing more to the public good (Fan, 2000; Sally \& Hill, 2006), Cipriani, Giuliano, and Jeanne (2007) found that younger children contributed more than older ones. At the same time, other work has shown that although older children initially contribute more, they also decrease contributions toward freeriding more readily than younger children (Harbaugh \& Krause, 2000). Yet, this work cannot clarify whether older children freeride more readily regardless of other players' strategies or, alternatively, whether they freeride mainly when others freeride, but would also cooperate more when others cooperate (conditional cooperation).

Second, inconsistent findings also abound in relation to gender. The literature on adults reports gender differences in opposite directions. Although some studies have shown that cooperation in PGGs is more characteristic of females, others have identified males as more cooperative (for a review see Croson \& Gneezy, 2009). Similar inconsistencies exist for children. Whereas Harbaugh and Krause (2000) found no significant gender effects on cooperative behavior over the course of the game, in a study by Vogelsang, Jensen, Kirschner, Tennie, and Tomasello (2014), boys freerode more than girls. In a study by Cipriani, Giuliano, and Jeanne (2007), girls also tended to show more cooperative behavior.

In part, these inconsistencies may be attributable to a lack of systematic control for strategic interdependence. In most work on children, experimenters group multiple subjects together, whose outcomes thus depend on each other's choices. To be sure, this approach may benefit ecological validity by exposing subjects to the "real-life" strategies of other co-players. Yet, greater ecological validity often entails some sacrifice of experimental control and causal inference. For example, the behavior of a child interacting with cooperative co-players is difficult to compare to the behavior of another child interacting with selfish co-players. Although some work with children has employed computergenerated co-players (Leipold, Vetter, Dittrich, LehmannWaffenschmidt, \& Kliegel, 2013; McClure et al., 2007; Sally \& Hill, 2006), these studies either implemented responsive algorithms that tethered the co-players' contributions to the previous decision of the subject (McClure et al., 2007; Sally \& Hill, 2006) or divided subjects into different experimental groups, with each subgroup facing different strategies (Leipold et al., 2013). Thus, little or no 
work has used experimental designs in which each subject faced identical strategies of the other co-players.

Additionally, the abstractness of most experimental designs (e.g., playing for money or tokens) arguably makes it harder for children to understand the strategic features of the situation, rendering cognitive development a potential confound. Of the few studies that have attempted to translate the PGG to a more concrete child-appropriate context (Alencar, De Oliveira Siqueira, \& Yamamoto, 2008; Vogelsang et al., 2014), setups have proven resource-intensive, and none have adopted a computerized methodology, which would vastly simplify data collection.

\section{Aims and hypotheses}

To address some of the limitations of previous work, the present study introduces a newly developed, age-appropriate computer task based on a concrete real-life situation, called the Pizzagame. We report data collected with this task in a sample of children and adolescents 9 to 16 years of age. In the Pizzagame, children are led to believe they are connected over the Internet with three different sets of two same-sex coplayers. All of the players receive a fixed set of resources (i.e., slices of pizza) that they can decide to pool toward the public good (i.e., take to school) or not (i.e., leave at home). At school, the "virtual teacher" adds $50 \%$ of all pooled slices that are then equally redistributed among all players.

In total, the Pizzagame progresses through three conditions in a predetermined sequence, each condition consisting of four rounds each. In each condition, the co-players, who are in fact computer-generated, follow fixed strategies. During the first condition, subjects face cooperative co-players who contribute high quantities of their resource to the public good. In the second condition, they interact with selfish co-players who only contribute very little, whereas, in the final third condition, co-players' strategies diverge, with one co-player exhibiting a cooperative and the other exhibiting a selfish strategy.

The present study pursued three main aims. The first and primary aim was to introduce and show the feasibility and reliability of a new life-like PGG that controls for the factor of strategic interdependency to assess cooperation and defection among children and adolescents. However, a mere description of the Pizzagame along with the claim that it is feasible and reliable would have begged the question as to whether the task is in fact a valid measure of cooperative behavior for children and adolescents.

As a second aim, we therefore sought to demonstrate the validity of this new measure. Given that prior findings indicate that individuals predominantly adopt a conditionally cooperative strategy, we expected that subjects would be cooperative toward cooperative, selfish toward selfish and show a medium level of contributions toward divergent co-players (Hypothesis 1).

Third, we aimed to shed further light on the role of age and gender effects in cooperative behavior to enrich our understanding of the developmental roots of cooperative behavior in children and adolescents. Therefore, we examined whether age and gender had a significant impact on two dimensions within the PGG, namely on contributions in each of the three different conditions and on behavioral change between conditions. Hence, as most studies report that older children show more cooperative behavior than younger children, we predicted that older children would contribute more across all three conditions (Hypothesis 2a). At the same time, on the basis of the finding that freeriding spreads more readily among older children (Harbaugh \& Krause, 2000), we predicted that older children would adapt more readily to the strategies of their coplayers. That is, with increasing age children would show more pronounced lowering of contributions toward selfish co-players (compared to cooperative co-players), but also more pronounced elevation of contributions toward the divergent co-players, who were moderately cooperative (Hypothesis 2b). With respect to possible gender effects on cooperative behavior it is not clear if they exist, but if they do, girls seem to be more cooperative than boys. Thus, we predicted that girls would show more cooperative behavior across all three conditions (Hypothesis 3). Regarding gender effects on behavioral change between conditions, there was no empirical evidence we could derive a testable hypothesis from. Thus, we explored the impact of gender on behavioral changes between conditions.

\section{Method}

\section{Sample}

We recruited 216 children and adolescents from 9 to 16 years of age as part of a general population sample of an ongoing large-scale study in a medium-sized German city (for detailed information, see White et al., 2015). Institutional review board (IRB) approval was obtained from the university ethics committee. Parents or legal guardians consented and youth assented after being informed about the study prior to participation.

To rule out that children misunderstood the strategic setup of the PGG, we asked comprehension questions (see below) during the training phase of the procedure. Accordingly, in our analyses, we excluded 23 subjects because they erred on two or more out of nine comprehension questions. Finally, the data from two subjects were not saved due to a technical error, yielding a final sample of 191 subjects ( $57.1 \%$ girls, $42.9 \%$ boys; $M_{\text {age }}=12.03$ years, $S D=1.92$ ). With the exception of 
the very few 16-year-olds, age was spread relatively evenly across the full range (see Fig. 1).

There were no significant age differences between girls and boys $\left[t_{\text {gender }}(189)=0.03, p=.97\right]$. Additionally, parental education and monthly household income did not differ as a function of age [parent education, $r_{\text {age }}(179)=-.07, p=.33$; monthly household income, $r_{\text {age }}(181)=.001, p=.99$ ] or gender [parent education, $t_{\text {gender }}(179)=0.20, p=.84$; monthly household income, $\left.t_{\text {gender }}(182)=-0.22, p=.83\right]$.

\section{Instructions and setup}

As part of the large-scale study, children were invited for one appointment that lasted approximately $3 \mathrm{~h}$. They received a battery of measures (e.g., a storytelling task, verbal skills test, and several questionnaires and interviews). The Pizzagame was the penultimate procedure of the appointment. Before starting the Pizzagame, subjects received thorough information about the rules and setup (i.e., number of trials, number of players, etc.) of the game via a slide show. They were also informed that the value of the gift they could choose at the end of the appointment would increase with the number of slices of virtual pizza they managed to retrieve throughout the course of the game. Such incentivization is a common feature of economic games, to ensure a basic degree of motivation among the subjects. Specifically, we used three boxes of different sizes (small, medium, and big), each of which contained a different set of gifts. We informed children that the biggest box contained the most attractive presents, the medium box contained moderately attractive presents, and the smallest box contained the least attractive presents. We also told them that the more slices they collected during the game, the bigger the box would be that they could choose a gift from (incentivization). Through this procedure, we aimed to prevent specific gift preferences from affecting the results. Unbeknownst to subjects, everyone was offered the same selection of presents from the big box, to not place anyone at a disadvantage due to their game behavior.

Instructions were followed by three illustrative scenarios (i.e., noncooperative, exploitative, and cooperative) showing different potential outcomes of the game. Multiple scenarios were used to safeguard against biasing children in their decision-making. To check subjects' comprehension of the strategic configuration of the PGG, they were asked the following questions regarding each scenario: "Which players have more pizza slices than at the start of the round?," "Which players have the same number of pizza slices as at the start of the round?," and "Which players have fewer pizza slices than at the start of the round?"

Afterward, the experimenters ran a test version of the game, to explain and familiarize subjects with the game interface. To further enhance the cover story, subjects were asked if they, just like their co-players, would be comfortable with a picture being taken of them via the webcam. In the absence of their child, parents were informed about the deception used in the PGG, and they consented to it and to all parts of the game before the procedure was started (see the Discussion section).

After starting the game, the experimenter claimed to have something else to do, took a seat at another table, and asked the child to continue playing the game. This aimed to minimize socially desirable response patterns due to the presence of the experimenter. The appointment was videotaped to check and ensure a high level of standardization throughout the period of data collection. Subjects' choices were recorded directly by the E-Prime software suite (Schneider \& Zuccoloto, 2007). After the Pizzagame, subjects evaluated

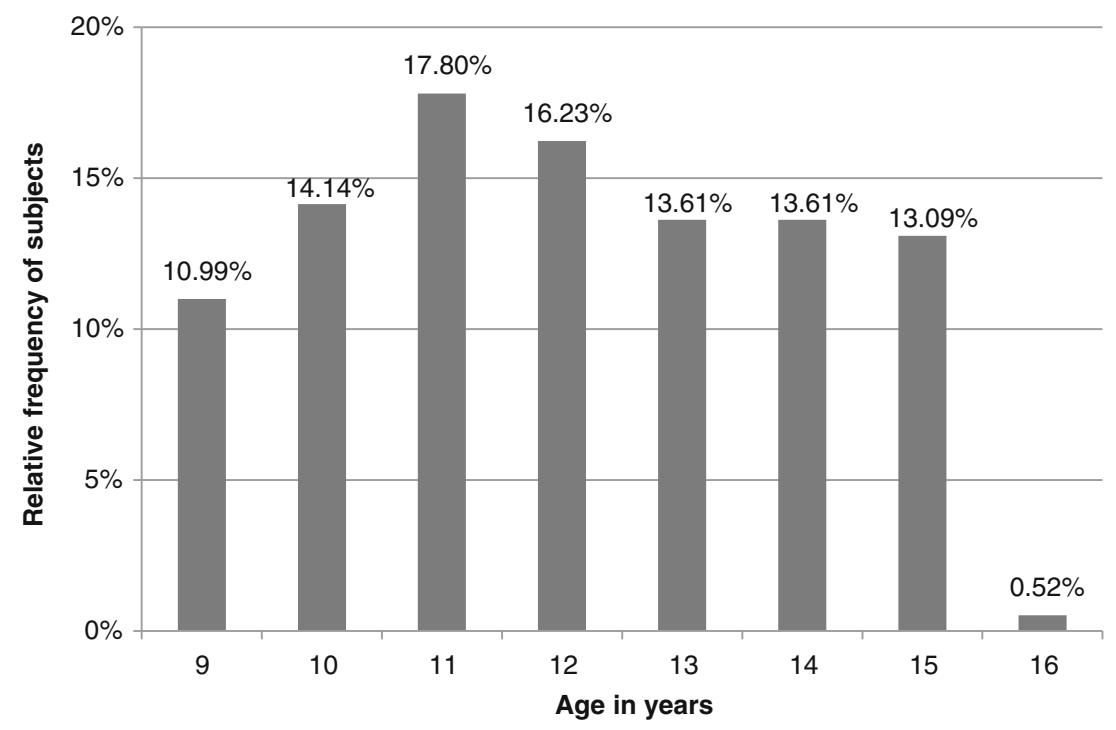

Fig. 1 Age distribution of subjects 
the appointment, including an open question asking what part of the appointment they had liked best (the question allowed children to name more than one measure as their favorite).

\section{Design of the Pizzagame}

The Pizzagame implements the structural features of a PGG. In the course of developing the Pizzagame, we aimed to find a scenario that was as close to a life-like situation as possible. In line with other peer-based paradigms (Crowley, Wu, Molfese, \& Mayes, 2010; Gunther Moor, Bos, Crone, \& van der Molen, 2014; Guyer, Choate, Pine, \& Nelson, 2012; Reijntjes, Stegge, Terwogt, Kamphuis, \& Telch, 2006), we presumed that a situation in which boys and girls ostensibly interacted with same-age, same-sex peers in a school setting would act as a familiar and ecologically valid cue to trigger children's everyday social behavior. The importance of these aspects is persuasively underscored by ample data showing that concrete, familiar, and relevant scenarios improve performance on a variety of cognitive tasks, even among adults (e.g., Sperber \& Girotto, 2002; Wason \& Shapiro, 1971), and facilitate earlier understanding among children (e.g., Doherty, 2009; Donaldson, 1978). Moreover, our use of pictures of other players, sound features, and background music for transitional slides built on previous adaptations of popular paradigms for children (e.g., Crowley et al., 2010) and were designed to intuitively appeal to children and adolescents. The Pizzagame was programmed and presented using the EPrime software suite (Schneider, Eschman, \& Zuccolotto, 2002). Before starting the game, children were informed about the rules of the game and led to believe that they were playing with two other children over the Internet by incorporating a fake website link. Actually, they played against computergenerated co-players with fixed strategies. We used this procedure to enhance the deception, in light of empirical evidence showing that people behave differently when they know they are interacting with computer agents than they do with humans (Krach et al., 2008; Shechtman \& Horowitz, 2003). This deception procedure draws on previous work using a similar strategy for the well-validated ballgame Cyberball (Crowley et al., 2010). To enhance the credibility of the cover story and minimize the impact of the subjects' inferences based on the different facial expressions of co-players, we used pictures from two emotional face databases of children with facial expressions confirmed as being neutral (Egger et al., 2011; Langner et al., 2010). To consolidate the impression that subjects were playing with three sets of two sameage, same-sex co-players, we used facial portraits of boys or girls 9 to 12 years of age for the younger subjects, and pictures of boys or girls 13 to 16 years of age for the older subjects.

Each of the four rounds of the different conditions of the Pizzagame begins by endowing the three players with nine virtual pizza slices. Without learning of one another's decisions, subjects ( $i$ for the subject, $j$ and $k$ for the preprogrammed coplayers) then decide how many slices (zero, three, six, or nine) they would prefer to leave at home (or keep for themselves), and how many they would like to bring to school and contribute to the public good $\left(g_{i}, g_{j}, g_{k}\right)$ (see Fig. 2A).

At school, all slices are placed on a "communal plate" (without showing which player contributed how much) before the virtual teacher adds $50 \%$ to whatever number of slices are on the plate (see Fig. 2B). Afterward, all slices on the plate are divided equally among the players, regardless of what each player contributed initially (see Fig. 2C). At the end of the round, the slices obtained at school and those left at home are added up to display the individual outcome of the round for subject $i$ (see Fig. 2D). The payoff per round and the overall payoff were displayed after each round (i.e., not permanently) to reduce the amount of information per screen and to minimize potential sources of distraction and confusion.

The payoff function that operationalizes the gains from each round for player $i$ is thus specified by the following equation: $\prod_{i}=9-g_{i}+0.5\left(g_{i}+g_{j}+g_{k}\right)$. The program performs all the computations in full view of the players to minimize the influence of mathematical competencies on game behavior.

In the first condition, subjects interact with highly cooperative co-players who both contribute all of their initial endowment of nine slices of pizza in the first round. In the subsequent three rounds, one co-player keeps on contributing nine slices, whereas the contributions of the other player slightly decline to six slices from the second round onward. In the second condition, the co-players pursue a selfish strategy, commencing with three and zero slices in the first round and then minimizing contributions to complete freeriding (zero slices) from the second round onward. Finally, in the third condition, the co-players adopt divergent strategies, with first-round contributions of nine and three slices from the cooperative and the selfish player, respectively. From the second round onward, the selfish player decreases the contributions toward complete freeriding, whereas the cooperative player carries on contributing all resources. The co-players' reductions of contributions from the first to the final round within each condition aimed to simulate the general behavioral pattern of decreasing contributions that was found in prior studies (e.g., Fehr \& Gächter, 2000; Harbaugh \& Krause, 2000).

\section{Data analysis}

First, we report descriptive statistics for all study variables using SPSS statistical software, version 20.0 (SPSS Inc). To demonstrate the feasibility of the instrument, we describe the number of errors on comprehension questions and the frequency with which children stated that the Pizzagame was their favorite part of the session, for the whole sample of 216 children (prior to applying exclusion criteria). Moreover, 


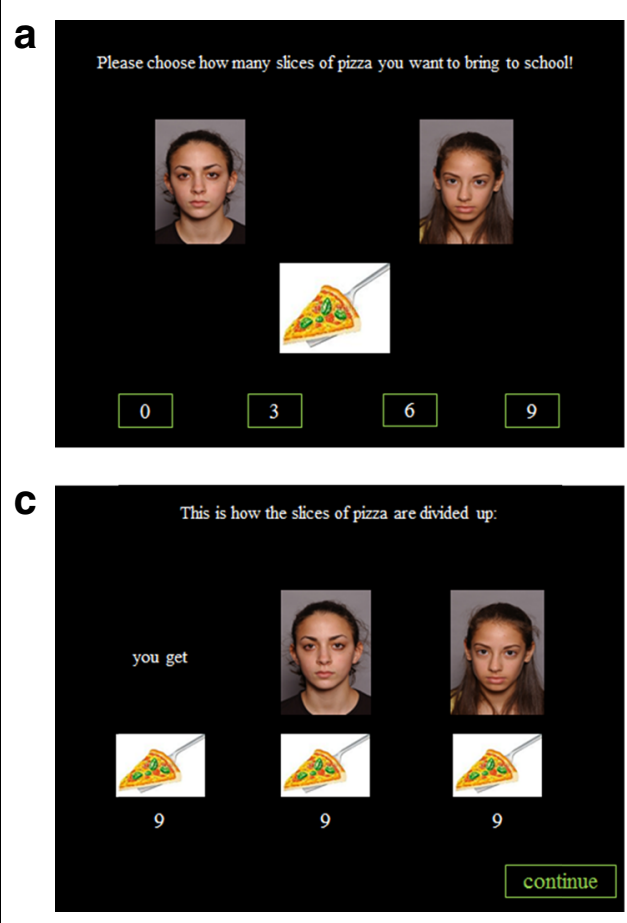

Fig. 2 One hypothetical round of the Pizzagame, illustrating the four key stages of the game: (A) Decision situation, with pictures of the co-players and the contribution options. (B) Display of anonymous individual contributions and subsidization by the teacher $(50 \%$ of the sum of the individual contributions). (C) Display of the redistribution of the public good

we report on the internal consistency of each condition by calculating Cronbach's alpha.

To test Hypotheses 1, 2a, 2b, and 3, we applied structural equation modeling (SEM) using Mplus 7.11 (Muthén \& Muthén, 2013). As Aguirre-Urreta (2014) pointed out, these techniques have several advantages, since they account for measurement error better and offer more complex models than traditional techniques, and might therefore strengthen data analysis in experimental research. These analyses were carried out in three steps:

Step 1 To test the first hypothesis regarding conditional cooperation, we compared differences in the latent mean contributions between conditions. To this end, a latent-state model was used to estimate the latent means of cooperative behavior in each of the three consecutive conditions. We specified a latent variable for each condition (i.e., cooperative, selfish, or divergent). The first round of each condition was not included in the analyses. The rationale behind this decision was that subjects could only incorporate information about the co-players' cooperative behavior into their decisions after playing the first round of each condition. Additionally, this minimized the potential

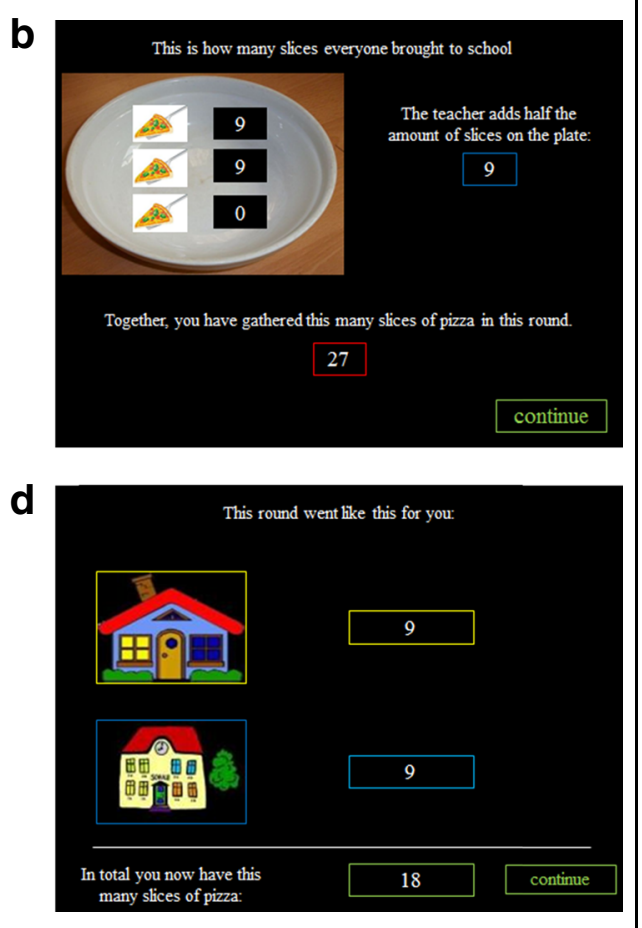

to each individual player. (D) Display of the resource balance after one round of the public goods game. The exemplary photographs in the figure are drawn from the NIMH Child Emotional Faces Picture Set (NIMHChEFS; Egger et al., 2011)

carryover of behavior from previous to subsequent conditions, reducing the risk of biasing the latent means. Accordingly, the last three rounds of each condition were used as indicators for the respective latent variables. No crossloadings were specified. We also specified an autocorrelated residual structure between the corresponding observed indicators from the three conditions (Sörbom, 1975). The latent mean scores were freely estimated using the effectcoding method for the identification of latent means (Little, 2013). To examine whether latent mean scores substantially differed between conditions, we specified three latent difference scores. To this end, we substracted the latent mean score of the selfish from that of the cooperative condition $(d 1)$, the latent mean score of the divergent from that of the selfish condition $(d 2)$, and the latent mean score of the divergent from that of the cooperative condition, and checked whether those differences in latent mean scores were significant.

Step 2 Age and gender were included in the latent-state model as time-invariant predictor variables to test our second hypothesis. All three latent variables 
were regressed on both age and gender, and we analyzed their impact on latent mean scores in all three conditions.

Step 3 As a third step, we expanded the latent-state model to an autoregressive model in order to analyze the potential change in conditional cooperativeness as a function of age and gender. Thus, autoregressive paths were specified from the latent mean scores of the cooperative to the selfish and from the selfish to the divergent condition. In this autoregressive model, we also analyzed the impact of age and gender on the latent means of the selfish and divergent conditions, since those latent variables reflect the behavioral change. The model fit was evaluated using (a) the chi-square statistic, (b) the comparative fit index (CFI), (c) the root-mean squared error of approximation (RMSEA), and (d) the standardized root-mean squared residual (SRMR). According to $\mathrm{Hu}$ and Bentler (1999), a RMSEA $\leq .05$ (.08), a CFI $\geq .95$ (.90), and a SRMR $\leq .05$ (.08) indicate a good (or, respectively, an adequate) model fit.

\section{Results}

The results showed that the majority of children answered all nine comprehension questions correctly or committed only a single error (see Fig. 3). The numbers of errors on the comprehension questions negatively correlated with age $[r-$ age $(214)=-.22, p<.001]$ but did not differ between boys and girls $\left(M_{\text {male }}=0.4, S D=0.76 ; M_{\text {female }}=0.56, S D=1.07\right)$ $[t(214)=-1.31, p=.19]$. The excluded children were significantly younger than the included children $\left(M_{\text {age excl }}=\right.$ $\left.11.00, S D=1.96 ; M_{\text {age }{ }_{\text {incl }}}=12.03, S D=1.92\right)[t$ incl excl $(214)=2.58, p<.05]$, but there was no gender difference between the excluded and included children, $\chi^{2}(1, N=$ $216)=1.09, p=.30$. Furthermore, $66.3 \%$ of the subjects stated that the Pizzagame was their favorite part of the appointment, followed by the verbal skills test $(19.3 \%)$ and the storytelling task (14.4\%).

In the first round of the cooperative condition, subjects contributed $3.09(34.3 \%)$ pizza slices. The first-round contributions of all conditions were not associated with age $[r$ coop $(191)=.064, p=.381 ; r_{\text {self }}(191)=.116, p=.111 ; r-$ $\operatorname{diverg}(191)=-.010, p=.889]$ and did not differ as a function of gender $\left[t_{\text {coop }}(189)=-0.330, p=.742 ; t_{\text {self }}(156.949)=\right.$ $\left.0.084, p=.993 ; t_{\text {diverg }}(189)=-0.581, p=.562\right]$. The distributions of contributions initially increased in the cooperative condition, followed by a decrease in the selfish condition, and a rise back to intermediate levels in the divergent condition (see Fig. 4).

The contributions in each condition yielded acceptable internal consistency (cooperative condition, $\alpha=.762$; selfish condition, $\alpha=.663$; divergent condition, $\alpha=.889$ ).

The latent-state model testing the first hypothesis, regarding conditional cooperation, showed an adequate model fit, $\chi^{2}(15)=30.047, p<.05$, CFI $=.973$, RMSEA $=.072$, SRMR $=.035$. The estimated means of the latent variables indicated that the level of cooperative behavior was highest toward cooperative co-players $\left(M_{\text {coop }}=4.02, S D=2.39\right)$, lowest toward selfish co-players $\left(M_{\text {self }}=1.77, S D=2.05\right)$, and at a medium level toward divergent co-players $\left(M_{\text {diverg }}=2.98\right.$, $S D=2.71$ ). Significant differences emerged between the latent means of the cooperative and selfish conditions ( $\mathrm{d} 1=$ $2.25, p<.001)$, between the selfish and divergent conditions $(\mathrm{d} 2=-1.22, p<.001)$, and between the cooperative and

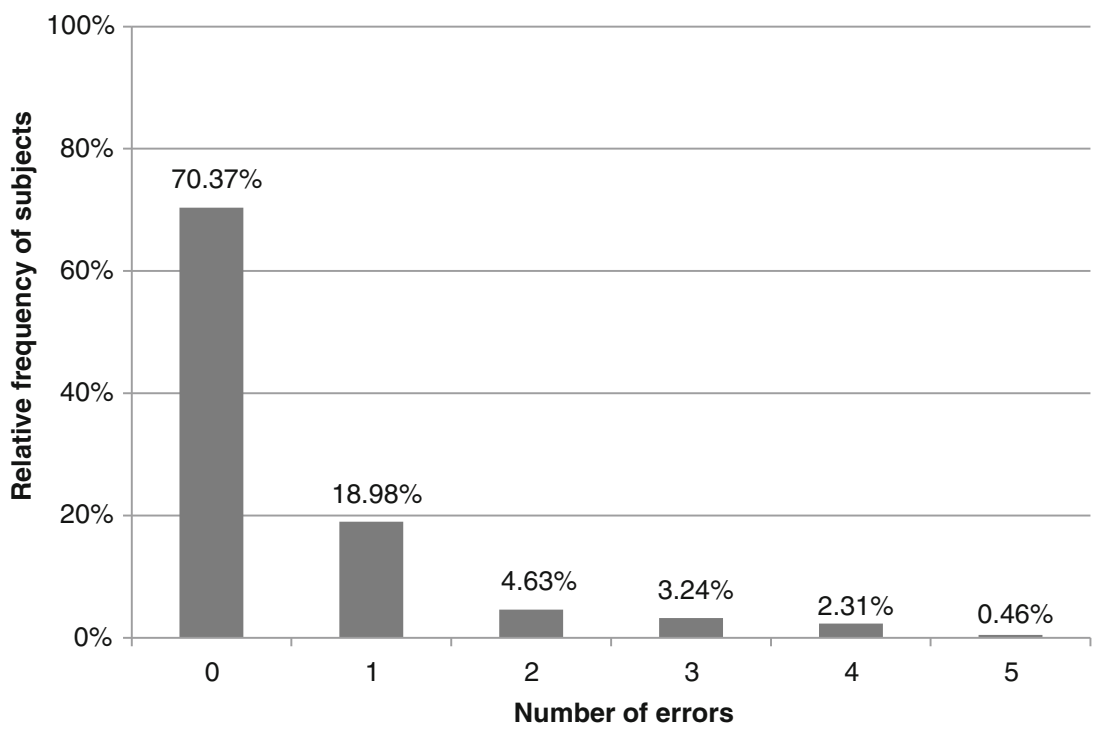

Fig. 3 Distribution of committed numbers of errors on the nine comprehension questions (no children committed more than five errors) 


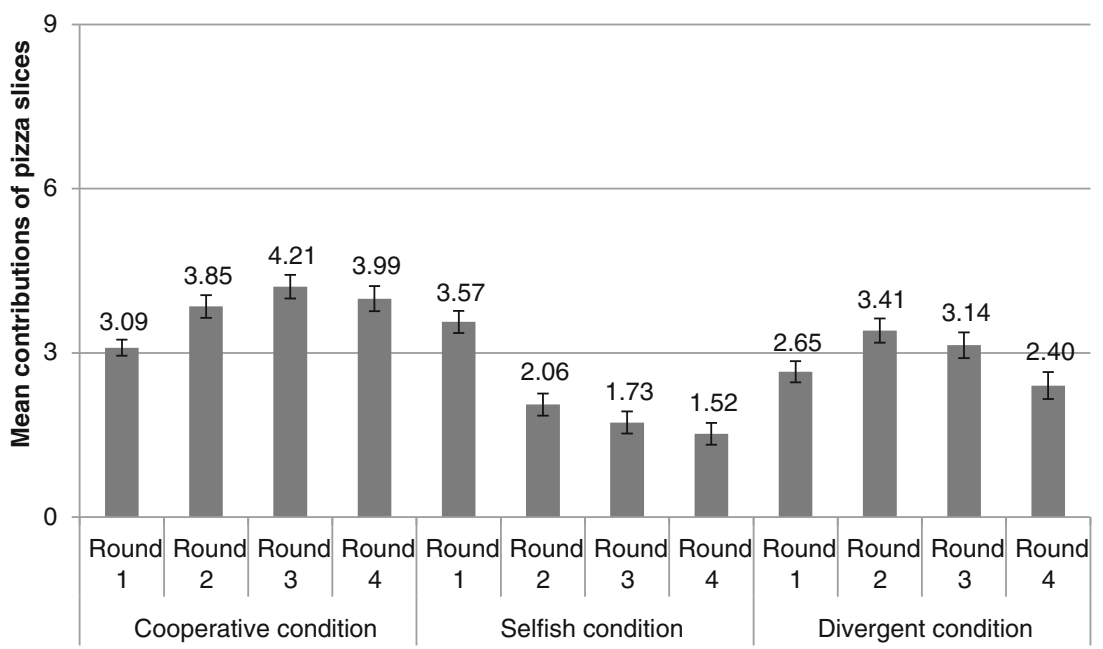

Rounds of the three conditions of the Pizzagame

Fig. 4 Mean contributions and the corresponding standard error bars of each round of the cooperative, selfish, and divergent conditions

divergent conditions $(\mathrm{d} 3=1.03, p<.001)$, indicating decreased contributions when co-players were selfish or divergent rather than cooperative, and increased contributions when co-players were divergent rather than selfish.

In a second step, age and gender were included in the latent-state model as time-invariant covariates, to test their impacts on the contributions (Hypotheses 2a and 3). The resulting model showed an adequate model fit, $\chi^{2}(27)=$ 41.694, $p<.05, \mathrm{CFI}=.975, \mathrm{RMSEA}=.053, \mathrm{SRMR}=.037$. The results revealed that neither age (cooperative condition, $\beta=.028, p=.741$; selfish condition, $\beta=-.156, p=.091$; divergent condition, $\beta=.064, p=.434$ ) nor gender (cooperative condition, $\beta=.087, p=.298$; selfish condition, $\beta=.081$, $p=.389$; divergent condition, $\beta=-.040, p=.622$ ) had an impact on the level of contributions across all three conditions.

An autoregressive model (see Fig. 5 was used to test whether age and gender predicted the change in contributions between conditions (Hypotheses 2b). The model showed an adequate model fit, $\chi^{2}(28)=41.700, p<.05$, $\mathrm{CFI}=.976$, RMSEA $=.051, \mathrm{SRMR}=.037$. The results indicated that children decreased their contributions from the cooperative to the selfish condition more readily with increasing age $(\beta=-.18, p<.05)$. Furthermore, they also increased their contributions more substantially from the selfish to the divergent condition with increasing age $(\beta=.21, p<.01$; see Fig. 6$)$. In contrast, gender impacted behavioral change neither from the cooperative to the selfish condition $(\beta=.002, p=.977)$ nor from the selfish to the divergent condition $(\beta=-.12, p=.107)$.

\section{Discussion}

Our study suggests that the Pizzagame - a newly developed, computerized, child-friendly PGG based on a concrete real-life scenario-is an engaging instrument to

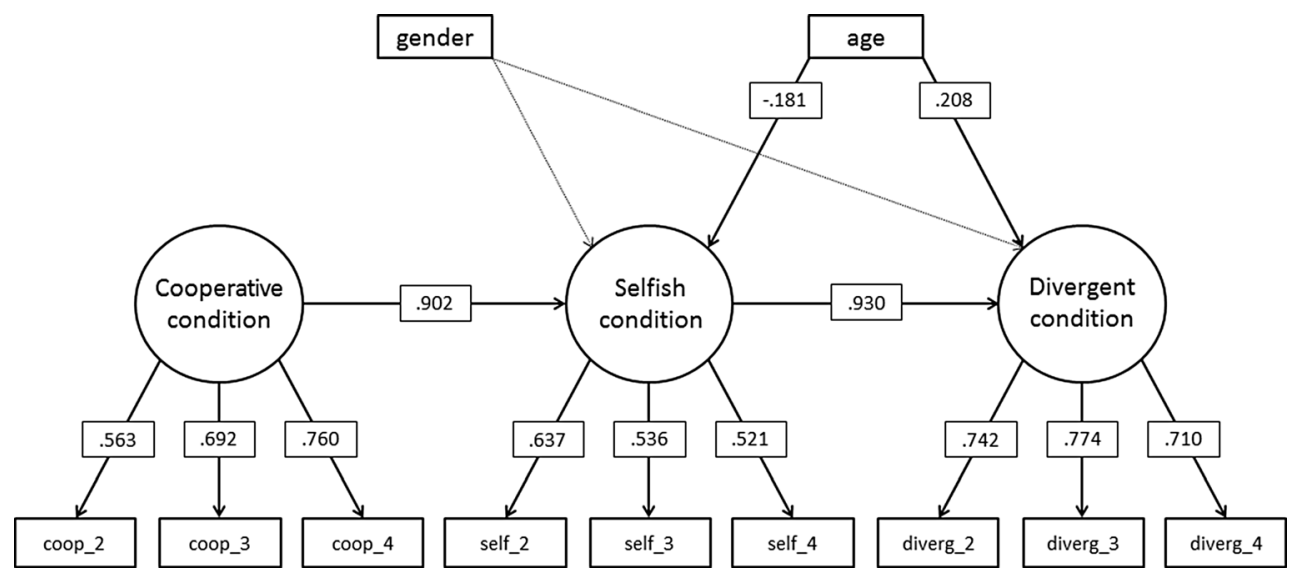

Fig. 5 Autoregressive model with standardized coefficients and paths between the three latent variables of the corresponding conditions (cooperative, selfish, and divergent) and age and gender as time-invariant predictor variables (dashed lines indicate nonsignificant paths) 


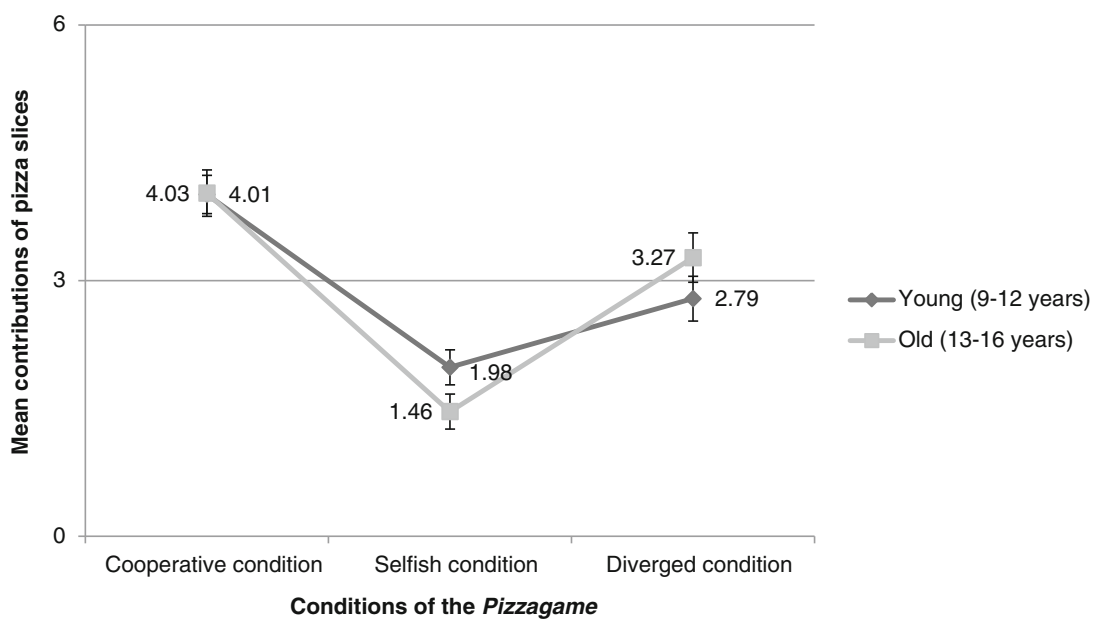

Fig. 6 Illustrative graph displaying the latent mean contributions and standard error bars of the cooperative, selfish, and divergent conditions for younger (9-12 years) and older (13-16 years) children. (Please note that subjects were grouped into younger and older subjects to illustrate the age effect for the purposes of this graph. All analyses were based on age as a continuous variable) feasibly and reliably assess the cooperative behavior of children and adolescents. Lending support to the validity of the Pizzagame, our pattern of results confirmed that children exhibit a conditionally cooperative strategy; that is, they cooperate when others do, but also act selfishly when others do. Intriguingly, we also found that this conditionally cooperative strategy varied as a function of age, such that cooperation became more conditional on other players' strategies as children transitioned to adolescence.

Regarding the feasibility of our task, the Pizzagame was by far the most engaging procedure of the appointment. This is especially remarkable considering that other procedures (e.g., the storytelling task) that are often considered highly engaging (Emde, Wolf, \& Oppenheim, 2003) were also part of the session. Besides this, our impression was that children often reacted quite emotionally when their co-players shifted their strategies, which additionally underscored the engaging nature of the Pizzagame.

Supporting the Pizzagame as a valid measure for children and adolescents, our results confirmed that we effectively altered the cooperative behavior of our subjects. The Pizzagame was therefore successful in evoking a behavioral pattern that followed a conditionally cooperative strategy, thus falling into line with other findings among both children (Vogelsang et al., 2014) and adults (Fischbacher et al., 2001).

The first-round contributions in our study (i.e., $34.3 \%$ ) fell just below the lower end of the typical range detected by other studies (40\%-60\%). In general, it is difficult to know why this minor discrepancy occurred, and future research might examine whether this result is inherent to the specifics of the Pizzagame or simply due to error variance. Potentially, the $33 \%$ choice ( 3 slices) may have struck the best balance between initial caution and still signaling a willingness to cooperate in the first round $(58.6 \%$ of children opted for the $33 \%$ choice in the initial round), giving rise to lower initial round contributions.

In contrast to our hypotheses, neither age nor gender had a significant direct impact on how much, on average, subjects contributed toward cooperative, selfish, or divergent co-players. However, our data yield support for an increasingly conditional strategy of cooperation as children transition toward adolescence. This raises an interesting alternative interpretation of previous findings that have mainly reported linear associations between age and cooperativeness. Rather than contributing more or less than younger children, older children may adapt more readily to both the cooperative and selfish strategies of their coplayers. Thus, developmental differences may emerge primarily when children face variations in the strategic behavior of their co-players that compel them to flexibly tailor their behavior accordingly. This could reflect a heightened sensitivity regarding the meaning of social behaviors and cooperative strategies among older children (e.g., due to better perspective-taking skills).

The null effects regarding gender agree with Harbaugh and Krause's (2000) study, but they contrast with other work on children (Cipriani et al., 2007; Vogelsang et al., 2014). Given that gender effects have emerged among both younger and older children (Cipriani et al., 2007; Vogelsang et al., 2014), a gender effect that diminishes with age is not likely. Another possible explanation for the absence of gender effects comes from a study on adults (Espinosa \& Kováŕík, 2015). The results of that study imply that when the context of the experiment is neutral, men and women do not behave differently. This may have also applied here, because we devised the Pizzagame interface to be as gender-neutral as possible. However, further research will be needed to clarify the role of gender in cooperative behavior in PGGs. 


\section{Limitations}

Some limitations deserve mentioning. First, and most importantly, the conditions were not counterbalanced to control for any order effects. For example, it is conceivable that individuals were influenced by the initial experience of cooperative co-players for the remainder of the game. For the present purposes, we opted against counterbalancing, due to the demands that this would have placed on the number of different sequential arrangements, as well as a balanced distribution of young and old girls and boys. Furthermore, because all subjects were exposed to all conditions, individual differences are still meaningful, even though the mean contributions might differ when reordering the sequence of conditions. As a first step toward validating the Pizzagame, and in line with other paradigms that use similar setups (e.g., trust-rupture-trust, inclusion-exclusion-inclusion; King-Casas et al., 2008; White, Wu, Borelli, Mayes, \& Crowley, 2013), we assumed that the cooperative-exploitative-divergent order would yield the most informative results. Specifically, we did not want children to face selfish co-players at the outset, because this might have had a frustrating effect on subjects at an early stage, as well as repercussions on engagement and carryover effects on later conditions. Moreover, we assumed that the best way to establish baseline cooperative behaviors was to program the co-players to begin cooperatively. By placing the exploitative strategy second, we aimed to induce a large behavioral change from the cooperative condition, whereas the divergent condition would tap into a potential recovery of cooperative behavior and offer subjects a choice between cooperative and exploitative strategies. Future research should certainly explore the order of the conditions.

Second, we used a forced choice design to make the game easier for children and adolescents. To be sure, a forced choice design makes comparisons with other studies on adults using open-choice paradigms tentative. At the same time, we faced a trade-off between comparability with previous work and the age appropriateness of the paradigm, and fell on the side of the latter given the main aim of this study.

Besides its use of forced choice, our paradigm may raise concerns whether children (especially the older ones) actually believed they were connected to real children over the Internet. Our impression from the video recordings, however, was that most children were very engaged with the task-for example, responding with negative affect to the shift from cooperative to selfish co-players. In addition, previous work using other social-interaction paradigms (e.g., Crowley et al., 2010; White et al., 2013) has also implemented similar measures while yielding valid and reliable results.

Finally, it is noteworthy that the Pizzagame involves deception. We believe that deception critically enhances the credibility and ecological validity of the Pizzagame, and it is therefore the preferred mode of administration (Bonetti,
1998). However, deception may raise ethical concerns (e.g., debriefing subjects). Importantly, debriefing may be less effective for younger children (e.g., due to difficulties in reappraising experience in light of new information), and may even induce children to distrust the experimenters and lead to negative affect (see Thompson, 1990, pp. 11-12). We therefore informed caregivers about the deception and then asked them (1) whether they consented to their child playing the Pizzagame and (2) whether they would like the experimenter to fully debrief their child afterward regarding the deception. Notably, all children were exposed to an uplifting closing experience in our procedure (the moderately cooperative condition followed by receiving a gift from the biggest box), to defuse potential negative affect. Also, they were given contact information of clinically trained personnel that they were encouraged to contact in the event of further distress. Overall, it is important to weigh the pros and cons of deception in the Pizzagame and to obtain ethical approval from the IRB before using deception.

\section{Conclusion}

We consider the Pizzagame to be a highly valuable tool for future research on cooperative behavior in children and adolescents, for a number of reasons. First, the paradigm has proven highly engaging for both children and adolescents. Second, as compared to social games, with multiple real-life subjects requiring coordination, the Pizzagame greatly simplifies data collection and the measurement of individual cooperative strategies while controlling for strategic interdependence. Third, using this instrument permits flexible manipulation of the strategies of co-players in various ways, thus allowing investigation of individual differences in cooperation in different contexts. Finally, and perhaps most importantly, the instrument enables objective assessment of developmental and individual differences in the cooperative behavior of children and adolescents. In so doing, the Pizzagame complements the vast number of subjective self-report measures for children, parents, and teachers to assess cooperative behavior of children (and similar constructs), commonly used in developmental science. Here, we have presented first evidence for a developmental shift toward more conditional cooperation as children move from middle childhood to adolescence. Given the burgeoning literature showing that peer problems figure prominently in the formation and maintenance of maladaptive behavior (Parker, Rubin, Stephen, Wojslawowicz, \& Buskirk, 2006), behavioral assessments of cooperative strategies applicable to large samples may also add an important layer of understanding to the field of developmental psychopathology.

Author note The preparation of this article and the research presented herein were supported by grantsfrom the German Federal Ministry of 
Education and Research (Grants 01KR1201A to E) as well as by LIFE Leipzig Research Center for Civilization Diseases, Universität Leipzig. LIFE is funded by means of the European Union, by the European Regional Development Fund (ERDF) and by means of the Free State of Saxony within the framework of the excellence initiative. The responsibility for the contents of this publication lies with the authors.

\section{References}

Aguirre-Urreta, M. (2014). Improving between-group comparisons in IS research through the use of SEM and latent variables: An introduction and some examples. Communications of the Association for Information Systems, 34, 30. Retrieved from http://aisel.aisnet. org/cais/vol34/iss1/30/

Alencar, A. I., De Oliveira Siqueira, J., \& Yamamoto, M. E. (2008). Does group size matter? Cheating and cooperation in Brazilian school children. Evolution and Human Behavior, 29, 42-48. doi:10.1016 /j.evolhumbehav.2007.09.001

Bonetti, S. (1998). Experimental economics and deception. Journal of Economic Psychology, 19, 377-395. doi:10.1016/S01674870(98)00012-9

Chaudhuri, A. (2011). Sustaining cooperation in laboratory public goods experiments: A selective survey of the literature. Experimental Economics, 14, 47-83. doi:10.1007/s10683-010-9257-1

Cipriani, M., Giuliano, P., \& Jeanne, O. (2007). Like mother like son? Experimental evidence on the transmission of values from parents to children (IZA Discussion Paper No. 2768). Retrieved from http://hdl.handle.net/10419/34481

Colman, A. M. (2003). Cooperation, psychological game theory, and limitations of rationality in social interaction. Behavioral and Brain Sciences, 26, 139-198. doi:10.1017/S0140525X03000050

Croson, R., \& Gneezy, U. (2009). Gender differences in preferences. Journal of Economic Literature, 47, 448-474.

Crowley, M. J., Wu, J., Molfese, P. J., \& Mayes, L. C. (2010). Social exclusion in middle childhood: rejection events, slow-wave neural activity, and ostracism distress. Social Neuroscience, 5, 483-495.

Dawes, R. M. (1980). Social dilemmas. Annual Review of Psychology, 31, 169-193. doi:10.1146/annurev.ps.31.020180.001125

De Dreu, C. K. (2013). Human cooperation: Challenges for science and practice. Psychological Science in the Public Interest, 14, 117-118. doi:10.1177/1529100613496959

Doherty, M. J. (2009). Theory of mind: How children understand others' thoughts and feelings. Hove: Psychology Press.

Donaldson, M. (1978). Children's minds. New York: Norton.

Egger, H. L., Pine, D. S., Nelson, E. E., Leibenluft, E., Ernst, M., Towbin, K. E., \& Angold, A. (2011). The NIMH Child Emotional Faces Picture Set (NIMH-ChEFS): A new set of children's facial emotion stimuli. International Journal of Methods in Psychiatric Research, 20, 145-156.

Emde, R. N., Wolf, D. P., \& Oppenheim, D. (2003). Revealing the inner worlds of young children: The MacArthur story stem battery and parent-child narratives. New York: Oxford University Press.

Espinosa, M. P., \& Kováŕík, J. (2015). Prosocial behavior and gender. Frontiers in Behavioral Neuroscience, 9, 88. doi:10.3389 /fnbeh.2015.00088

Fan, C.-P. (2000). Teaching children cooperation-An application of experimental game theory. Journal of Economic Behavior and Organization, 41, 191-209. doi:10.1016/S0167-2681(99)00072-4

Fehr, E., \& Gächter, S. (2000). Cooperation and punishment in public goods experiments. American Economic Review, 90, 980-994.

Fischbacher, U., Gächter, S., \& Fehr, E. (2001). Are people conditionally cooperative? Evidence from a public goods experiment. Economics Letters, 71, 397-404. doi:10.1016/S0165-1765(01)00394-9
Gross, M., \& Heinrichs, H. (2010). Environmental sociology: European perspectives and interdisciplinary challenges. Dordrecht, The Netherlands: Springer.

Gummerum, M., Hanoch, Y., \& Keller, M. (2008). When child development meets economic game theory: An interdisciplinary approach to investigating social development. Human Development, 51, 235 261. doi: $10.1159 / 000151494$

Gunther Moor, B., Bos, M. G. N., Crone, E. A., \& van der Molen, M. W. (2014). Peer rejection cues induce cardiac slowing after transition into adolescence. Developmental Psychology, 50, 947-955. doi: $10.1037 / \mathrm{a} 0033842$

Guyer, A. E., Choate, V. R., Pine, D. S., \& Nelson, E. E. (2012). Neural circuitry underlying affective response to peer feedback in adolescence. Social Cognitive and Affective Neuroscience, 7, 81-92.

Harbaugh, W. T., \& Krause, K. (2000). Children's altruism in public good and dictator experiments. Economic Inquiry, 38, 95-109.

Hardin, G. (1968). The tragedy of the commons. Science, 162, $1243-$ 1248. doi:10.1126/science.162.3859.1243

Hu, L., \& Bentler, P. M. (1999). Cutoff criteria for fit indexes in covariance structure analysis: Conventional criteria versus new alternatives. Structural Equation Modeling, 6, 1-55. doi:10.1080 /10705519909540118

King-Casas, B., Sharp, C., Lomax-Bream, L., Lohrenz, T., Fonagy, P., \& Montague, P. R. (2008). The rupture and repair of cooperation in borderline personality disorder. Science, 321, 806-810.

Krach, S., Hegel, F., Wrede, B., Sagerer, G., Binkofski, F., \& Kircher, T. (2008). Can machines think? Interaction and perspective taking with robots investigated via fMRI. PLoS ONE, 3, e2597. doi:10.1371 /journal.pone.0002597

Langner, O., Dotsch, R., Bijlstra, G., Wigboldus, D. H. J., Hawk, S. T., \& van Knippenberg, A. (2010). Presentation and validation of the Radboud Faces Database. Cognition and Emotion, 24, 1377-1388. doi:10.1080/02699930903485076

Ledyard, J. O. (1995). Public goods: A survey of experimental research. In J. H. Kagel \& A. E. Roth (Eds.), The handbook of experimental economics (chap. 2). Princeton, NJ: Princeton University Press.

Leipold, K., Vetter, N. C., Dittrich, M., Lehmann-Waffenschmidt, M., \& Kliegel, M. (2013). Individual and developmental differences in the relationship between preferences and theory of mind. Journal of Neuroscience, Psychology, and Economics, 6, 236-251. doi:10.1037/npe0000008

Little, T. D. (2013). Longitudinal structural equation modeling. Methodology in the social sciences. New York: Guilford Press.

McClure, E. B., Parrish, J. M., Nelson, E. E., Easter, J., Thorne, J. F., Rilling, J. K.,...Pine, D. S. (2007). Responses to conflict and cooperation in adolescents with anxiety and mood disorders. Journal of Abnormal Child Psychology, 35, 567-577. 10.1007/s10802-0079113-8

Muthén, L. K., \& Muthén, B. (2013). Mplus statistical software (Version 7.11). Los Angeles: Muthén \& Muthén.

Olson, M. (1965). The logic of collective action: Public goods and the theory of groups. Cambridge: Harvard University Press.

Parker, J. G., Rubin, K. H., Stephen, E. A., Wojslawowicz, J. C., \& Buskirk, A. A. (2006). Peer relationships, child development, and adjustment: A developmental psychopathology perspective. In D. Cicchetti \& D. J. Cohen (Eds.), Developmental psychopathology: Theory and method (pp. 419-493). New York: Wiley.

Parks, C. D., Joireman, J., \& Van Lange, P. A. (2013). Cooperation, trust, and antagonism: How public goods are promoted. Psychological Science in the Public Interest, 14, 119-165. doi:10.1177 /1529100612474436

Reijntjes, A., Stegge, H., Terwogt, M. M., Kamphuis, J. H., \& Telch, M. J. (2006). Emotion regulation and its effects on mood improvement in response to an in vivo peer rejection challenge. Emotion, 6, 543552. doi:10.1037/1528-3542.6.4.543 
Sally, D., \& Hill, E. (2006). The development of interpersonal strategy: Autism, theory-of-mind, cooperation and fairness. Journal of Economic Psychology, 27, 73-97. doi:10.1016/j.joep.2005.06.015

Schneider, W., Eschman, A., \& Zuccolotto, A. (2002). E-Prime computer software and manual. Pittsburgh: Psychology Software Tools Inc.

Schneider, E., \& Zuccoloto, A. (2007). E-Prime 2.0 [Computer software]. Pittsburg: Psychological Software Tools.

Shechtman, N., \& Horowitz, L. M. (2003). Media inequality in conversation: How people behave differently when interacting with computers and people. In Proceedings of the SIGCHI conference on Human factors in computing systems (pp. 281-288). New York: ACM.

Sörbom, D. (1975). Detection of correlated errors in longitudinal data. British Journal of Mathematical and Statistical Psychology, 28, 138-151.

Sperber, D., \& Girotto, V. (2002). Use or misuse of the selection task? Rejoinder to Fiddick, Cosmides, and Tooby. Cognition, 85, 277290.

Thompson, R. A. (1990). Vulnerability in research: A developmental perspective on research risk. Child Development, 61, 1-16.
Van Lange, P. A., Joireman, J., Parks, C. D., \& van Dijk, E. (2013). The psychology of social dilemmas: A review. Organizational Behavior and Human Decision Processes, 120, 125-141.

Vogelsang, M., Jensen, K., Kirschner, S., Tennie, C., \& Tomasello, M. (2014). Preschoolers are sensitive to free riding in a public goods game. Frontiers in Psychology, 5, 729. doi:10.3389 /fpsyg.2014.00729

Wason, P. C., \& Shapiro, D. (1971). Natural and contrived experience in a reasoning problem. Quarterly Journal of Experimental Psychology, 23, 63-71.

White, L. O., Klein, A. M., Kirschbaum, C., Kurz-Adam, M., Uhr, M., Müller-Myhsok, B.,...Stalder, T. (2015). Analyzing pathways from childhood maltreatment to internalizing symptoms and disorders in children and adolescents (AMIS): A study protocol. BMC Psychiatry, 15, 126

White, L. O., Wu, J., Borelli, J. L., Mayes, L. C., \& Crowley, M. J. (2013). Play it again: Neural responses to reunion with excluders predicted by attachment patterns. Developmental Science, 16, 850-863.

Zelmer, J. (2003). Linear public goods experiments: A meta-analysis. Experimental Economics, 6, 299-310. 